Results Observational studies showed reduction of speeds. Total number of road traffic crashes reduced in Naivasha after introducing speed radar camera. Fatal cases increased in 2011 comparing with 2009 and 2010. Serious and slight injury cases reduced in 2011 comparing with 2009 and 2010. In Thika, total number of cases as well as (severity of cases increased during 2011 comparing with 2009 and 2010. The amount of fines collected from the offenders increased to about Kenya Shillings(Ksh) 3000000.

Significance Findings of this study can be the thinking-point for future intervention considering speed control, injury prevention and increase in revenue.

\title{
22 EFFECTS OF SPEED RADAR CAMERA IN REDUCING ROAD TRAFFIC CRASHES: EXPERIENCES FROM KENYA
}

doi:10.1136/injuryprev-2012-04059022

DK Kibogong, SM Chowdhury. World Health Organization-Kenya Country Office, Kenya

Background Road traffic injuries are among the top 10 causes of death accounting for $2.1 \%$ of total global mortality. Excess and inappropriate speed is responsible for a high proportion of the mortality and morbidity that result from road crashes. Over speeding is one of the major risk factors in Kenya.

Objectives The purpose of this study was to investigate the effects of speed radar camera in reducing road traffic injuries.

Methods Data on road traffic crashes was collected from Naivasha and Thika district from the police department between April and December 2012 after using speed radar cameras by the police officers. Data from the police department for last 3 years were analysed to compare with the data after introducing speed radar camera. 